\title{
REGRAS PARA NOMENCLATURA DOS NOMES COMUNS DOS AGROTÓXICOS
}

\author{
ROSÂNGELA BLOTTA ABAKERLI * \\ ELISABETH FRANCISCONI FAY ** \\ PETER REMBISCHEVSKI *** \\ ANA MARIA VEKIC **** \\ KENIA GODOY ***** \\ ADRIANA DE ARAÚJO MAXIMIANO ****** \\ ARLINDO BONIFÁCIO *******
}

\begin{abstract}
Este trabalho visou subsidiar as ações da Agência Nacional de Vigilância Sanitária (ANVISA) na padronização da nomenclatura dos ingredientes ativos usados em agrotóxicos, segundo normas internacionais. As regras apresentadas resultam do trabalho de revisão da ortografia dos nomes comuns dos agrotóxicos em uso no Brasil, publicados na "Relação de monografias dos ingredientes ativos de agrotóxicos, domissanitários e preservantes de madeira" da ANVISA (2002), conforme Resolução № 347/02. Foram utilizados os princípios gerais de atribuição de nomes comuns publicados pela International Standards Organization (ISO) e as regras de nomenclatura de compostos orgânicos. $O$ artigo não pretende esgotar a discussão sobre esse tema, sugerindo a padronização da ortografia dos nomes comuns dos agrotóxicos para facilitar o uso da informação pelos técnicos das áreas acadêmica, comercial e governamental.
\end{abstract}

* Química, PhD, EMBRAPA Meio Ambiente, Jaguariúna, SP, Brasil (e-mai: abakerli@cnpma.embrapa.br).

** Farmacêutica Bioquímica, MsC, EMBRAPA Meio Ambiente, Jaguariúna, SP, Brasil (e-mail: bethfay@cnpma.embrapa.br).

*** Químico, Gerência Geral de Toxicologia, Agência Nacional de Vigilância Sanitária, Brasília, DF, Brasil. (e-mail: peter.rembischevski@anvisa.gov.br).

**** Química, Especialização em Entomologia e Toxicologia, Gerência Geral de Toxicologia, Agência Nacional de Vigilância Sanitária, Brasília, DF, Brasil (e-mail: ana.vekik@anvisa.gov.br).

**** Química, MsC, Ministério do Meio Ambiente, Esplanada dos Ministérios, Brasília, DF, Brasil (e-mail: kenia.godoy@mma.gov.br).

***** Química, Diretoria de Licenciamento e Qualidade Ambiental, Instituto Brasileiro do Meio Ambiente, Brasília, DF, Brasil (e-mail: adriana.maximiano@ibama.gov.br).

*******Engenheiro Agrônomo, Coordenadoria de Fiscalização de Agrotóxicos, Ministério da Agricultura Pecuária e Abastecimento, Esplanada dos Ministérios, Brasília, Brasil (e-mail: arlindo@agricultura.gov.br). 


\section{INTRODUÇÃO}

Os nomes químicos dos ingredientes ativos usados nas formulações de agrotóxicos seguem as regras de nomenclatura de compostos orgânicos da International Union of Pure and Applied Chemistry (IUPAC) ou do Chemical Abstracts (CA). Contudo esses nomes químicos são complexos, extensos e difíceis de serem utilizados em linguagem coloquial. Assim, os ingredientes ativos recebem nomes comuns que são de propriedade pública e internacionalmente aceitos.

Os princípios para atribuição de nomes comuns aos agrotóxicos são descritos na norma 257 da ISO (1988). O nome comum, por definição, constitui nome livre para uso geral na identificação de uma substância química sem que seja necessário recorrer ao seu nome científico. Mais de mil ingredientes ativos já tiveram seus nomes comuns aprovados pela International Standards Organization (ISO), norma 1750 (ISO, 1981) e suas atualizações publicadas em 1982, 1983, 1999 e 2001.

Outros organismos de padronização, tais como o British Standards Institute (BSI), o American National Standards Institute (ANSI) e o Japanese Ministry of Agriculture Forestry and Fisheries (JMFA) também atribuem nomes comuns aos ingredientes ativos desenvolvidos nos respectivos países. A aprovação desses nomes pela ISO pode ser lenta, mas de modo geral são aceitos, desde que atendam os princípios da ISO 257 (1988). Os membros da ISO pertencem aos organismos nacionais de padronização de 130 países.

O objetivo da nomenclatura é fornecer nome curto, único e não-ambíguo, de fácil pronúncia e grafia, visando facilitar a comunicação e sua utilização conveniente nas esferas científica, comercial e governamental. Como tais nomes não são protegidos por patentes, não é permitido que se tornem marca registrada de produto idêntico ou semelhante. No Brasil alguns produtos comerciais receberam o mesmo nome do ingrediente ativo, o que deve ser evitado.

A padronização internacional dos nomes comuns dos agrotóxicos é efetuada pelo Comitê Técnico 81 (CT 81) da ISO. Seu trabalho consiste em assegurar que haja consistência entre o nome atribuído e a representatividade da estrutura química da substância, sem que exista muita proximidade com 0 nome puramente químico e que possa ser pronunciado apropriadamente. Outra preocupação do Comitê é evitar que haja coincidência com marcas de produtos comerciais existentes ou que conflitem com os nomes dos produtos farmacêuticos, possibilitando que o nome aprovado possa ser universalmente utilizado. A padronização dos nomes comuns de compostos para uso veterinário ou medicamentos não se enquadra nos objetivos da ISO/TC 81, esses são cobertos pelo International Nonproprietary Names for Pharmaceutical Substances mantido pela Organização Mundial de Saúde.

Os princípios de seleção do nome comum são poucos e muito simples. Merece citação o que leva em consideração a sua abrangência internacional, ou seja, que a identidade do nome possa ser mantida em todas as línguas, porém sujeita às modificações lingüísticas que se fizerem necessárias. Outro princípio descrito na norma ISO 257 (1988), que demonstra a preocupação em facilitar a tradução e ortografia dos nomes comuns refere-se à utilização da letra $f$ no lugar do $p h$. A exceção para esse princípio ocorre com o sufixo -phenyl nos nomes dos ésteres.

Da mesma forma que há critérios para a tradução dos nomes químicos para o português, os nomes comuns também devem seguir regras para evitar a desqualificação da língua portuguesa.

O objetivo deste documento é resumir as regras que devem ser aplicadas para efetivar a tradução (do inglês para o português) dos nomes comuns dos ingredientes ativos em uso no Brasil, bem como daqueles que posteriormente venham a ser registrados.

\section{MATERIAL E MÉTODOS}

Efetuou-se levantamento dos ingredientes ativos registrados no Brasil (ANVISA, 2003), aos quais foram aplicadas as regras de tradução para o português da nomenclatura de compostos orgânicos (FERNANDES et al., 2002). O trabalho baseou-se também nas normas de atribuição de nomes comuns 
aos ingredientes ativos (ISO, 1988) e nas regras básicas de ortografia do português do Brasil.

Os princípios para as denominações internacionais dos nomes comuns dos agrotóxicos descritos na norma 257 (ISO, 1988), baseiam-se nas seguintes regras gerais:

a) os nomes comuns dos ingredientes ativos devem ser distinguidos entre si fonética e ortograficamente, evitando-se também, os excessivamente longos;

b) o nome comum deverá indicar seu parentesco com outros compostos do mesmo grupo de atividade e origem química por meio de afixos, infixos e ou radicais;

c) devem ser evitados nomes que por ortografia ou fonética induzam confusão com outros já em uso;

d) os nomes comuns são considerados substantivos comuns e, portanto, escritos em letras minúsculas nos textos em geral, com exceção do início de frases e monografias;

e) a atribuição do nome comum é dada à molécula mãe quando esta possibilitar a existência de mais de uma substância. Os ingredientes ativos são apresentados em lista por ordem alfabética e seguidos por seus respectivos derivados, igualmente em ordem alfabética (por exemplo, fentina é a substânciamãe e seus derivados: acetato de fentina e hidróxido de fentina).

f) os nomes comuns devem ser o mais curto possível e não constituídos de siglas e/ou números, embora exceção seja feita para as substâncias bem conhecidas em que a utilização de outros nomes geraria confusão. Alguns nomes comuns atribuídos antes da elaboração dessa norma utilizavam letras e/ou números. Se os nomes consistirem nas siglas da substância, devem ser escritos em maiúsculas e sem utilização de espaço, como por exemplo o MCPA. Já para nomes com letras e números, os números devem ser separados por vírgula e as letras por hífem, como em 2,4,5-T e 2,4-D;

g) não são utilizados afixos para substâncias que existem em formas enantioméricas. O nome comum é atribuído à primeira solicitação independentemente de tratar-se de racemato ou enantiômero. Caso apareça, posteriormente, uma variação estereoquímica, o nome original é acrescido dos sufixos -MP, -M ou -P para o racemato, isômero (-) ou isômero (+), respectivamente (exemplo, metalaxil e metalaxil$\mathrm{M})$.

As regras gerais para a tradução dos nomes comuns dos agrotóxicos para o português do Brasil envolvem:

a) o nome em português é a tradução do nome aprovado e publicado pela ISO 1750 (1981, 1982, 1983, 1999, 2001), mantendo-se o máximo possível a fonética original e adequando-se a ortografia ao vernáculo (por exemplo, aldicarb e aldicarbe);

b) o gênero do nome do agrotóxico é vinculado à classe química ou nome químico conforme as regras de nomenclatura da IUPAC;

c) consoantes mudas no final do nome, ou seja, os nomes terminados em -b; -c; - $d$; - $p$; - t; e outras consoantes deverão ser seguidas por vogais;

d) utilizar as letras -f-, -t-e -c-no lugar de -ph-,-th- $e$-ch-respectivamente;

e) não utilizar a letra $-n$ no final da palavra;

f) não usar a letra - $h$ - entre vogais;

g) trocar a letra $-y$ - pela letra $-i-$;

h) somente utilizar $-m$ antes de -b ou $-p$;

i) usar -rr-entre vogais quando a fonética da letra $r$ for forte (erre);

j) usar - $r$ - entre vogais quando a fonética da letra $r$ for fraca (ere);

k) usar -ss-entre vogais quando a fonética da letra $s$ for forte (esse);

l) usar -s-entre vogais quando a fonética da letra $s$ for a da letra $z$.

\section{RESULTADOS E DISCUSSÃO}

A Tabela 1 resume a adequação ortográfica dos afixos e infixos encontrados nos nomes comuns dos ingredientes ativos, sendo fornecidos exemplos. 
TABELA 1 - ADEQUAÇÃO DA ORTOGRAFIA DE AFIXOS E INFIXOS DOS NOMES COMUNS

\begin{tabular}{|c|c|c|c|}
\hline \multicolumn{2}{|c|}{ Afixos e infixos } & \multicolumn{2}{|c|}{ Exemplos } \\
\hline Portuguas & InglOs & Portuguas & InglOs \\
\hline$-b-$ & -bb- & Feido giberØlico & gibberellic acid \\
\hline C- & k- & casugamicina & kasugamicin \\
\hline \multirow[t]{2}{*}{$-\mathrm{C}-$} & $-\mathrm{ch}-$ & rincoforol & rhynchoforol \\
\hline & -chlor- & diclorv s & dichlorvos \\
\hline$C-$ & chlor- & clorotalonil & chlorothalonil \\
\hline cum- & coum- & cumacloro & coumachlor \\
\hline -cum & -coum & brodifacum & brodifacoum \\
\hline es- & S- & espinosade & spinosad \\
\hline$f-$ & ph- & fosmete & phosmet \\
\hline$-f-$ & -ph- & alfa-cipermetrina & alpha-cypermethrin \\
\hline hi- & hy- & hidrazida maleica & maleic hydrazide \\
\hline$-1-$ & $-\|-$ & aletrina & allethrin \\
\hline$-m p-$ & $-n p-$ & etofemproxi & etofenprox \\
\hline$-n f-$ & $-m f-$ & etrinf $s$ & etrimfos \\
\hline$-n s-$ & $-\mathrm{ms}-$ & foranssulfurom & foramsulfuron \\
\hline qu- & ch- & quinometionato & chinomethionat \\
\hline$-r-$ & $-r-$ fraco & forato & phorate \\
\hline$-r r-$ & $-r$ - forte & biorresmetrina & bioresmethrin \\
\hline$-s-$ & -s- fraco & flumetsulam & flumetsulam \\
\hline -SS- & -s- forte & flazassulfurom & flazasulfuron \\
\hline$-t-$ & -th- & clortal & chlorthal \\
\hline
\end{tabular}

Nota:

zzz-: significa que o termo é um prefixo e estará posicionado no início da palavra;

-zzz-: significa que o termo é um infixo e estará posicionado no meio da palavra;

-zzz: significa que o termo é um sufixo e estará posicionado no final da palavra.

Um dos princípios estabelecidos pelo CT 81 (ISO) envolve as substâncias cujos nomes químicos já são razoavelmente curtos e não-ambíguos, não havendo necessidade de atribuição de nomes comuns como, por exemplo, brometo de metila, metaldeído e outros. A listagem dessas substâncias, apesar de não atualizada, está publicada na norma 765 da ISO (1976). Nessa situação encontram-se as substâncias descritas na Tabela 2, cuja tradução dos seus nomes segue os critérios já conhecidos para tradução de nomes químicos do inglês para o português (FERNANDES et. al., 2002).

Na ausência de nomenclatura ISO deverá ser utilizado o nome aprovado por outras organizações de padronização (como o BSI, o ANSI, o JMAF), a qual deve ser indicada a fim de evitar variações e multiplicidade de nomes para a mesma substância. O JMAF utiliza siglas para muitos nomes comuns dos ingredientes ativos.

Conforme já descrito, o nome comum das substâncias sugere uma relação com sua estrutura química (por exemplo, o nome glifosato é composto de gli- de glicina, -fos- de fosfono e -ato que é a terminação de sal). Essa lógica foi mantida, dentro do possível, para a tradução dos sufixos dos nomes comuns dos princípios ativos descritos na Tabela 3. Considerando que a denominação deve manter relação com a funcionalidade química, as exceções descritas na Tabela 3 decorrem da inexistência de grupo funcional cetona nas estruturas dos compostos. Pelos princípios da ISO 257 (1988) não é admissível utilizar nos nomes comuns sufixos que induzam conclusão errônea da estrutura química da substância. Nessa situação encontram-se os compostos diazinom, dissulfotom dalapom e fosfamidom. Por outro lado, vários ingredientes ativos apresentam sufixos nos nomes para os quais haverá forte desvirtuação de sua fonética original ao se adotar simplesmente o critério de correlação estrutural. As substâncias com sufixo -carb, que se refere ao grupo carbamato, teriam a rigor a tradução 
-carbo para se manter o gênero da função química, ou -carba para representar o grupo. Nessa situação foi dada prioridade à manutenção da fonética com simples adequação da ortografia -carbe. Esses casos de manutenção da fonética original estão sintetizados na Tabela 4, a qual também resume os sufixos que permanecem inalterados em relação aos nomes originais em inglês (como por exemplo, os terminados em -dim).

\section{TABELA 2 - SUBSTÂNCIAS QUE NÃO REQUEREM NOME COMUM ISO}

\begin{tabular}{|c|c|}
\hline \multicolumn{2}{|c|}{ Exemplos } \\
\hline Portuguas & InglOs \\
\hline Aeido b rico & boric acid \\
\hline Aeido cian drico & hydrogen cyanide \\
\hline ÆĖido giberØico & gibberellic acid \\
\hline Aeido indolbut rico & indolbutyric acid \\
\hline AtEcool laur lico & lauryl alcohol \\
\hline bicarbonato de pot/Assio & potassium bicarbonate \\
\hline borax & borax \\
\hline brometo de metila & methyl bromide \\
\hline cianamida & cyanamide \\
\hline cinetina & kinetin \\
\hline codlelure & codlelure \\
\hline decanol & n-decanol \\
\hline enxofre & sulfur \\
\hline fosfeto de alum nio & aluminium phosphide \\
\hline fosfeto de magnøsio & magnesium phosphide \\
\hline hidrazida maleica & maleic hydrazide \\
\hline hidr xido de cobre & copper hydroxide \\
\hline metalde do & metaldehyde \\
\hline oxicloreto de cobre & copper oxychloride \\
\hline xido cuproso & cuprous oxide \\
\hline xido de tributilestanho & tributyltin oxide \\
\hline pentaclorofenol & pentachlorophenol \\
\hline pentaclorofen xido de s dio & $\begin{array}{c}\text { sodium pentachlorophenoxide } \\
\text { pvrethrin }\end{array}$ \\
\hline sulfato de cobre & conner sulfate \\
\hline tribromofenol & tribromophenol \\
\hline
\end{tabular}

Conforme recomendação na norma 257 (ISO, 1988), os compostos que são sais quaternários de nitrogênio devem conter, forçosamente, o sufixo -quat. A rigor a tradução seria -quato, em razão dos compostos serem sais. No entanto, sugere-se a manutenção da fonética com simples adequação ortográfica -quate.

As terminações -ac e -ic representam um ácido e descrevem essa característica do ingrediente ativo. Dessa maneira, poder-se-ia recomendar o sufixo - aco. Entretanto, optou-se pela manutenção da fonética-aque e-ique, como, por exemplo, quincloraque e imazapique.

Os sufixos -fen referem-se ao grupo fenila dos éteres difenílicos, preferindo-se a manutenção da fonética com terminação em -fem. Em situação diversa o sufixo -ben passa a ser grafado como beno, sugerindo a presença do grupo benziltio- na molécula.

Diversos ingredientes ativos são substâncias com caráter ácido ou básico, que podem existir 
em grande variedade de sais ou ésteres. Quando a atividade biológica é devida à molécula-mãe (ácido ou base), os nomes comuns dos ingredientes ativos são atribuídos aos ácidos, alcoóis e bases-mãe, como cialofope (ácido-mãe), dinosebe (álcool-mãe) ou diquate (base-mãe). Nos ésteres e sais dessas substâncias, os radicais ou cátions e ânions complementares são indicados com modificadores na grafia dos seus nomes.

TABELA 3 - TRADUÇÃO DOS SUFIXOS DOS NOMES COMUNS COM BASE NA SUA CARACTERÍSTICA QUÍMICA

\begin{tabular}{|c|c|c|c|c|}
\hline \multicolumn{2}{|c|}{ Sufixos } & \multicolumn{2}{|c|}{ Exemplos } & \multirow[t]{2}{*}{ Exce I es } \\
\hline Portuguas & Ingl|as & Portuguas & Ingl|as & \\
\hline -alina & -alin & trifluralina & trifluralin & \\
\hline \multirow[t]{2}{*}{-amida } & -amid, & sulfluramida, & sulfluramid, & \\
\hline & -amide & niclosamida & niclosamide & \\
\hline -anilida & -anilide & ciclanilida & cyclanilide & \\
\hline -ano & -ane & lindano & lindane & \\
\hline \multirow[t]{2}{*}{-ato } & -at & quinometionato & chinomethionat & \\
\hline & -ate & fluvalinato & fluvalinate & \\
\hline -azina & -azine & atrazina & atrazine & \\
\hline -azol & -azole & etridiazol & etridiazole & \\
\hline -conazol & -conazole & propiconazol & propiconazole & \\
\hline -beno & -ben & piridabeno & pyridaben & \\
\hline -cloro & -chlor & butacloro & butachlor & \\
\hline -eno & -ene & quintozeno & quintozene & \\
\hline -furano & -furan & carbofurano & carbofuran & \\
\hline \multirow{3}{*}{-ona } & $\begin{array}{l}\text {-ite } \\
\text {-one }\end{array}$ & propargito & propargite & dalanom diazinom \\
\hline & $-0 m e$ & clomazona, & cromazone & dissulfotom, fosfamidom \\
\hline & -on & norflurazona & norflurazon & \\
\hline$-0 x i$ & $-0 x$ & bifenoxi & bifenox & \\
\hline -prido & -prid & acetamiprido & acetamiprid & \\
\hline -tiona & -thion & metidationa & metidathion & \\
\hline -trina & -thrin & resmetrina & resmethrin & \\
\hline & -tryn & ametrina & ametryn & \\
\hline -zida & -zide & tebufenozida & tebufenozide & \\
\hline
\end{tabular}

Para os sais ou ésteres de ácidos, o modificador é um sufixo hifenizado com sua ortografia na forma de adjetivo, como por exemplo cialofope-butílico ou fosetil-alumínico.

Para os ésteres de alcoóis e para os sais das bases, o nome do radical ou do ânion complementar é escrito como uma palavra separada e acrescida da preposição de (por exemplo: acetato de dinosebe, dibrometo de diquate).

Alguns íons e radicais apresentam nomes químicos que são muito complexos para compor o nome comum. Para aqueles de ocorrência mais freqüente têm sido desenvolvidos e recomendados nomes mais curtos para serem utilizados ao invés dos nomes químicos (Tabela 5). 
TABELA 4 - TRADUÇÃO DOS SUFIXOS DOS NOMES COMUNS COM BASE NA MANUTENÇÃO DA FONÉTICA ORIGINAL

\begin{tabular}{|c|c|c|c|}
\hline \multicolumn{2}{|c|}{ Sufixos } & \multicolumn{2}{|c|}{ Exemplos } \\
\hline Portuguas & Inglass & Portuguas & Inglas \\
\hline -al & -al & clortal & chlorthal \\
\hline$-a m$ & -am & metam & metam \\
\hline -ape & -ap & cartape & cartap \\
\hline -aque & $-a c$ & quincloraque & quinclorac \\
\hline -ar & -ar & acibenzolar & acibenzolar \\
\hline$-a z$ & $-a z$ & amitraz & amitraz \\
\hline -carbe & -carb & aldicarbe & aldicarb \\
\hline- de & $-d$ & nalede & naled \\
\hline -dim & $-\operatorname{dim}$ & clefoxidim & clefoxydim \\
\hline -ebe & $-e b$ & zinebe & zineb \\
\hline -ete & -et & folpete & folpet \\
\hline -fem & -fen & lactofem & lactofen \\
\hline -fom & -fon & tetradifom & tetradifon \\
\hline -fom & -phon & triclorfom & trichlorphon \\
\hline -fope & -fop & quizalofope & quizalofop \\
\hline$-f s$ & -fos & profenof $s$ & profenofos \\
\hline$-f s$ & -phos; & bromof $s$ & bromophos \\
\hline$-i l$ & -il; -yl & imazalil; metalaxil & imazalil; metalaxyl \\
\hline$-\mathrm{im}$ & $-i m$ & foxim & phoxim \\
\hline -ique & -ic & imazapique & imazapic \\
\hline$-m$ & $-n$ & $\begin{array}{c}\text { captam, dalapom, } \\
\text { diazinom, dicloram, } \\
\text { diflufenicam, dissulfotom, } \\
\text { fosfamidom }\end{array}$ & $\begin{array}{c}\text { captan, dalapon, } \\
\text { diazinon, dichloran, } \\
\text { diflufenican, disulfoton, } \\
\text { phosphamidon }\end{array}$ \\
\hline -metom & -meton & prometom & prometon \\
\hline -morfe & -morph & tridemorfe & tridemorph \\
\hline -prope & -prop & fenoxaprope & fenoxaprop \\
\hline -quate & -quat & diquate & diquat \\
\hline -sulfam & -sulfan & endossulfam & endosulfan \\
\hline -urom & -uron & linurom & linuron \\
\hline$-V \quad s$ & -vos & diclorv s & dichlorvos \\
\hline
\end{tabular}

\section{TABELA 5 - NOMES RECOMENDADOS PARA DESCREVER ÍONS E RADICAIS EM SAIS E ÉSTERES DOS AGROTÓXICOS}

\begin{tabular}{|c|c|c|}
\hline $\begin{array}{c}\text { Nome } \\
\text { Recomendado }\end{array}$ & Nome qu mico & Exemplo \\
\hline albesila to & a lquilbenzenosulfonato & trialbesilato de iminoctadina \\
\hline butometil & 2 -butoxi-1-metiletil & fluroxipir-butom et lico \\
\hline butotil & 2 -butoxie til & diclorprope-butot lico \\
\hline diclexina & diciclohexilam nio & diclexinato de dinex \\
\hline dimolamina & (2-hidroxietil)dimetilam nio & oxapirazona-dimolam nico \\
\hline diolam in a & bis (2-hidroxietil)am nio & 2,4 -D -diolam nico \\
\hline etadil & 1,2 -etanodiil & TCA-etad lico \\
\hline e to til & 2 -e toxietil & haloxifope-etot lico \\
\hline isoctil & iso-octyl & MCPA-isoct lico \\
\hline meptil & 1 -m e tilhe ptil & fluroxipir-mept lico \\
\hline mexil & 1 -metilhexil & cloquintoceto-mex lico \\
\hline olamina & (2-hidroxietil)am nio & clopiralida-olam nico \\
\hline propargil & 2-propinil & clorazifope-proparg lico \\
\hline tefuril & (tetrahidro-2-furanil)metil & quizalofope-P-tefur lico \\
\hline trim Øsio & trim etilsulf nio & glifosa to -trim Øsico \\
\hline trolamina & tris (2-hidroxietil)am nio & $2,4,5$-T-trolam nico \\
\hline
\end{tabular}

Fonte: WOOD (2003). 


\title{
4 CONCLUSÃO
}

Foram apresentadas as normas para tradução dos infixos e afixos dos nomes comuns dos ingredientes ativos dos agrotóxicos. As regras existentes para as traduções dos nomes químicos de substâncias orgânicas foram mantidas e utilizadas, sem prejuízo da fonética e ortografia. Não obstante, este artigo não pretende esgotar a discussão sobre esse tema, mas busca sugestões de critérios racionais para a nomenclatura dos ingredientes ativos utilizados e registrados no país.

Sugere-se a padronização da ortografia desses nomes, facilitando sobremaneira o uso da informação, satisfazendo as exigências de utilidade e precisão e, que sejam amplamente utilizadas por técnicos das áreas acadêmica, comercial e governamental.

\begin{abstract}
RULES FOR NOMENCLATURE OF PESTICIDES COMMON NAMES

This work sought to subsidize the actions of the Agência Nacional de Vigilância Sanitária (ANVISA) in the standardization of the nomenclature of the active ingredients used in pesticides, according to international norms. The presented rules result of the work of revision of the spelling of the common names of the pesticides in use in Brazil, published in the "Relação de monografias dos ingredientes ativos de agrotóxicos, domissanitários e preservantes de madeira" of ANVISA (2002), according to Resolution N. 347/02. The general principles of attribution of common names published by International Standards Organization (ISO) and the rules of nomenclature of organic compounds were used. The article doesn't intend to drain the discussion on that theme, suggesting the standardization of the spelling of the common names of the pesticides to facilitate the use of the information for the technicians of the academic, commercial and government areas.

KEY-WORDS: PESTICIDES-NOMENCLATURE; PESTICIDES-COMMON NAMES.
\end{abstract}

\section{REFERÊNCIAS}

1 ANVISA. Consulta Pública no 50. 09 de junho de 2003. Diário Oficial [da] República Federativa do Brasil, Brasília, 11 jun. 2003. Disponível em: <http://www.anvisa.gov.br/base/visadoc/CP/CP[4882-2-0].PDF>. Acesso em: 04 nov. 2003.

2 FERNANDES, A. C.; HEROLD, B.; MAIA, H.; RAUTER, A. P.; RODRIGUES J. A. R. G. Guia IUPAC para a nomenclatura de compostos orgânicos. Tradução portuguesa nas variantes européia e brasileira de A guide to IUPAC nomenclature of organic compounds recommendations. Lisboa: LIDEL- Edições Técnicas, 2002.

3 ISO. International Standards Organization. Pesticides and other agrochemicals: principles for the selection of common names. Geneva, 1988. (International Standard, 257)

4 ISO. International Standards Organization. Pesticides considered not to require common names. Geneva, 1976. (International Standard, 765).

5 ISO. International Standards Organization. Pesticides and other agrochemicals: common names. Geneva, 1981. (International Standard, 1750).

6 ISO. International Standards Organization. Pesticides and other agrochemicals: common names, Amendment 1. Geneva, 1982. (International Standard, 1750).

7 ISO. International Standards Organization. Pesticides and other agrochemicals: common names, Addendum 1. Geneva, 1983. (International Standard, 1750).

8 ISO. International Standards Organization. Pesticides and other agrochemicals: common names, Addendum 2. Geneva, 1983. (International Standard, 1750).

9 ISO. International Standards Organization. Pesticides and other agrochemicals: common names, Amendment 2. Geneva, 1999. (International Standard, 1750).

10 ISO. International Standards Organization. Pesticides and other agrochemicals: common names, Amendment 3. Geneva, 2001. (International Standard, 1750).

11 WOOD, A. Compendium of pesticides common names. Disponível: http://www.hclrss.demon.co.uk/index.html. Acesso em: 22 jul. 2003. 